\title{
Ni Hollow Nanospheres: Preparation and Catalytic Activity
}

\author{
Kaifu Zhong, Pu Jin, and Qianwang Chen \\ Hefei National Laboratory for Physical Science at Microscale, Department of Materials Science \& Engineering, \\ School of Chemistry and Materials, University of Science and Technology of China, Hefei 230026, China
}

Received 1 November 2005; Revised 18 April 2006; Accepted 4 May 2006

A method to prepare monodispersed silica nanospheres as templates for fabrication of nickel-silica composite hollow spheres is presented. The structures for both silica nanospheres and nickel-silica composite hollow spheres were characterized by X-ray diffraction (XRD), transmission electron microscopy (TEM), and scanning electron microscopy (SEM). The catalytic activity for acetone hydrogenation on nickel-silica composite hollow spheres was evaluated, and high conversion efficiency of $70 \%$ with good selectivity of $82.7 \%$ to 2-propanol was observed. The mechanism of high catalytic activity and good selectivity in acetone hydrogenation reaction was discussed.

Copyright (c) 2006 Kaifu Zhong et al. This is an open access article distributed under the Creative Commons Attribution License, which permits unrestricted use, distribution, and reproduction in any medium, provided the original work is properly cited.

\section{INTRODUCTION}

It is well known that properties of materials inherently associate with their morphologies and sizes. Shape and size of tunable materials are desirable for many applications, such as catalysis, medicine, electronics, ceramics, pigments, and cosmetics [1-6].

Recently, monodispersed nanospheres with the unique chemical and physical properties have attracted much attention. Specifically, silica nanospheres have been extensively studied, as they can be easily prepared and simply modified to dope selectively or attach functional groups. Recent researches on monodispersed silica nanospheres are expanding rapidly to assemble nanospheres in two-dimensional (2D) and three-dimensional (3D) ordered superstructures, such as preparation of photonic crystals [7]. A key requirement to successfully produce such spatially ordered structure is the preparation of highly monodispersed silica nanospheres. In addition, silica nanospheres have wide practical applications in labeling technology, [8] separation technologies [9], and biomedicine. For the purpose of achieving specific physical, chemical, or biological performance, monodispersed nanospheres are often used as templates to prepare core-shell nanostructures or hollow nanospheres. The common way to prepare hollow spheres is to encapsulate nanospheres such as silica or polystyrene spheres with nanocrystals, followed by removal of the templates by utilizing acid or base etching $[10,11]$.

In the previous work, we have reported a coating process to prepare nickel-silica composite hollow nanospheres
(650 $\mathrm{nm}$ in outer diameter) with controllable shell thickness in large-scale. The shell with porous structure consists of needle-like nickel nanoparticles with an average size of $15 \mathrm{~nm}$ [12]. It has been reported that particle dimension of metal catalyst strongly influences catalytic activity and selectivity [13]. In order to investigate the activity and selectivity of nickel-silica composite hollow nanospheres in heterogeneous catalysis reaction, we have performed acetone hydrogenation reaction using the as-prepared catalyst at temperatures ranging from 180 to $220^{\circ} \mathrm{C}$, which exhibits good catalysis and high selectivity. In this paper, we further discuss the improvement of preparing monodispersed silica nanospheres and silica-nickel nanocomposite hollow spheres, the results of acetone hydrogenation, and its reaction and our explanation of the reaction mechanism.

\section{EXPERIMENTAL}

\subsection{Preparation of monodispersed silica nanospheres}

We have synthesized monodispersed silica nanospheres by tetraethyl orthosilicate (TEOS) hydrolysis as reported elsewhere [12]. The method to prepare silica nanospheres is not exactly the same as reported by Stobber et al. [14]. They used the gaseous ammonia saturated ethanol as solvent to control concentration of ammonia, while $\mathrm{H}_{2} \mathrm{O}$ was added later. In our experiment, we chose aqueous ammonia solution as the source for ammonia and added TEOS solution dropwise into the aqueous ammonia. The main advantage of this method is to simplify the preparation of base reagent 


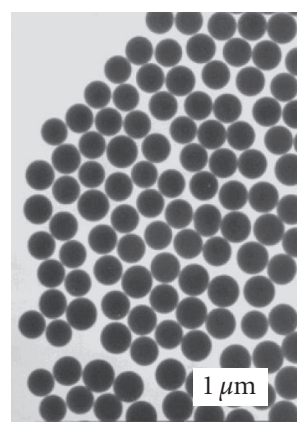

(a)

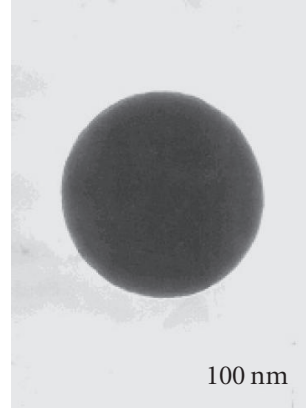

(b)

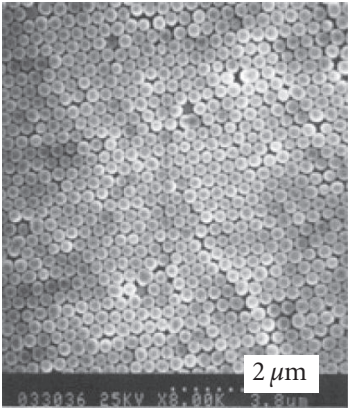

(c)

FIgURE 1: (a), (b) TEM images of bare silica nanospheres with a diameter of approximately $500 \mathrm{~nm}$, (c) SEM image of monodispersed silica nanospheres, which is in good agreement with the observation of TEM.

(ammonia solution) and keep the same concentration of aqueous ammonia. The adverse factor is the extra addition of water, which has a great influence on the nucleation and growth of silica nanospheres. During the experiment, we also have investigated the influence of main reagents on preparation of monodispersed silica nanospheres by changing concentrations of ammonia and TEOS.

\subsection{Preparation of $\mathrm{SiO}_{2} / \mathrm{Ni}_{3} \mathrm{Si}_{2} \mathrm{O}_{5}(\mathrm{OH})_{4}$ core-shell structures and nickel-silica hollow spheres}

The simple description of preparation of nickel-silica hollow spheres, the characterization of samples such as X-ray fluorescence analysis, dimethyl glyoxime gravimetric analysis, X-ray diffraction (XRD), transmission electron microscopy (TEM), scan electron microscopy (SEM), $\mathrm{N}_{2}$ adsorption/desorption isotherm data, and pressure composition measurement (PCT) have been reported elsewhere [12]. Samples were analyzed by Shimadzu XRF-1800 (40 kv $95 \mathrm{~mA}$ ) X-ray fluorescence spectrometer. XRD patterns were recorded using a Rigaku (Japan) D/max- $\gamma$ A X-ray diffractometer equipped with graphite monochromatized $\mathrm{Cu}$ Kalpha radiation $(\lambda=1.54178 \AA)$. SEM, TEM images, and electron diffraction (ED) were taken on Hitachi X-650 SEM and Hitachi H-800 TEM. Nitrogen adsorption isotherms of samples were measured by an ASAP 2010 volumetric sorption analyzer with an additional option for micropore analysis.

In this section, in order to find out the optimal way to prepare nickel-silica hollow spheres, we have investigated the influences of reaction order, morphology, and performance of samples through a series of controlled experiments. The process of coating nickel shells on silica templates was achieved by using homogeneous precipitation of slowly decomposing urea in nickel nitrate solution [12]. In order to better understand the reaction, we carried out the same reaction in the solution system without any silica nanosphere, at the same time we kept concentration of reagents, time, and temperature of reaction unchanged. The composition and phase of samples were analyzed by XRD. In addition, we used commercial silica nanospheres on micron scale as templates to prepare core-shell nanostructures, characterized morphology, and relevant properties of final samples compared to that of using the as-prepared silica nanospheres.

\subsection{Acetone hydrogenation on nickel-silica hollow nanospheres}

Acetone hydrogenation at 100,150 , and $200^{\circ} \mathrm{C}$ with silicasupported $\mathrm{Pd}$, nickel-silica composite hollow spheres, and Pd-precipitated composite hollow spheres was carried out [12]. Emphasis has been placed on selectivity of catalysts at $200^{\circ} \mathrm{C}$ and mechanism of acetone hydrogenation using nickel-silica hollow spheres.

\section{RESULTS AND DISCUSSION}

\subsection{Synthesis of monodispersed silica nanospheres}

As shown in Figures 1(a) and 1(b), silica nanospheres having uniform size and spherical morphology have been synthesized. Preferably monodispersed nanospheres with diameter of approximately $500 \mathrm{~nm}$ can be obtained through hydrolysis and polycondensation of TEOS. The direct SEM observation of the surface of silica nanosphere samples is shown in Figure 1(c), which is in good agreement with TEM images in Figures 1(a) and 1(b).

It has been suggested that ammonia as catalyst has an ability of controlling the morphology of silica nanospheres [14]. It is very important to keep constant concentration of ammonia in the overall process in order to synthesize monodispersed silica nanospheres. We studied the different sequences of adding reagents: adding ammonia dropwise into TEOS system or adding TEOS dropwise into ammonia system. Figure 2 shows SEM images of silica nanospheres obtained from choosing different sequences of adding reagents. The results showed that the first method was not as good as the second method to prepare monodispersed silica nanospheres. Two possible reasons might explain the phenomenon: one is that the first step of synthesizing silica 


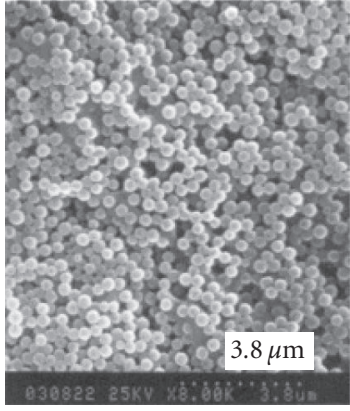

(a)

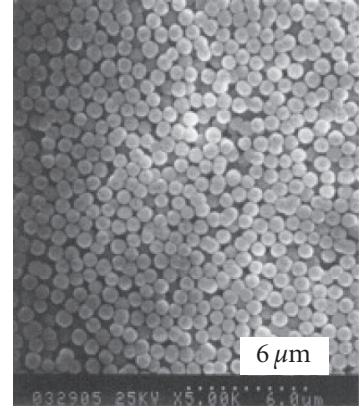

(b)

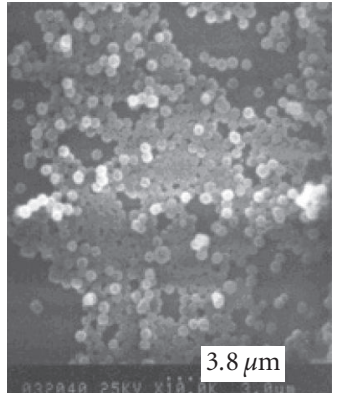

(a)

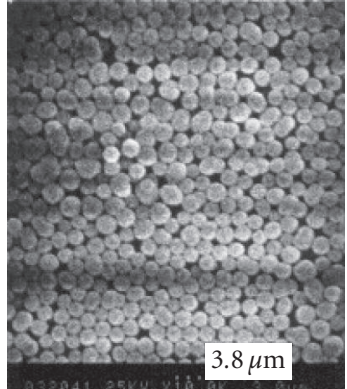

(b)

FIGURE 2: SEM images of silica nanospheres obtained from different sequences of adding reagents: (a) from adding ammonia dropwise into TEOS system; (b) from adding TEOS dropwise into ammonia system.

spheres is hydrolysis of TEOS. Adding ammonia into TEOS system inevitably brings some amount of water, which greatly affects the rate of TEOS hydrolysis, and finally results in the multidispersion of final particles. The other possible interpretation is ammonia's ability of controlling morphology of nanoparticles. In the first method, concentration of ammonia in the system ranges greatly at different stages of the reaction, resulting in different abilities of controlling the shape of silica nanoparticles. This leads to the appearance of the elliptical and other irregular shapes of silica nanoparticles. We also found that it is not easy to control the ratio of reagents, adjust the parameters to the optimal condition, and control the size and uniformity of particles when we choose the first method. At the same time, it is easy to gelate by forming silica network structure, and finally unable to obtain the regular shape of nanoparticles. In the second method, the variation of ammonia concentration is relatively low when adding TEOS into the system. Therefore, the second method is more preferable for synthesizing the monodispersed silica nanospheres.

In order to investigate the influence of reagent ratio, we used different concentrations of ammonia and TEOS. First, ammonia with a concentration of $1 \mathrm{~mol} / \mathrm{L}$ was introduced to the TEOS system, which resulted in a very slow hydrolysis of TEOS. The solution system finally became an azury silica sol with irregular particles. When using high ammonia concentration of $25 \%$ and $28 \%$, spherical silica particles were obtained. This indicated the critical catalytic effect of ammonia on hydrolysis of TEOS and controlling morphology of silica nanospheres. Second, we altered the concentration of TEOS while remaining other conditions unchanged. Figure 3 shows SEM images of silica nanospheres obtained from using different concentrations of TEOS in the reaction system of $20 \mathrm{ml}$. We observed that the size of silica nanospheres increase with increasing TEOS concentration. In addition, nonspherical particles became more and more with increasing TEOS concentration. The appearance of irregular silica particles could be explained by different growth rates of particles during the reaction.

\subsection{Preparation of $\mathrm{SiO}_{2} / \mathrm{Ni}_{3} \mathrm{Si}_{2} \mathrm{O}_{5}(\mathrm{OH})_{4}$ core-shell structures and nickel-silica hollow nanospheres}

In the control experiment, XRD profile of samples obtained from decomposing urea in nickel nitrate solution without any silica nanosphere at a constant temperature of $95^{\circ} \mathrm{C}$ is given in Figure 4(a). XRD analysis indicates that the precipitate is poor crystalline hexagonal $\alpha-3 \mathrm{Ni}(\mathrm{OH})_{2} \cdot \mathrm{xH}_{2} \mathrm{O}$ (JCPDS no. 22-0444), with a growth orientation at (001). Figure 4(b) shows thermal decomposition result of the precipitates from 0 to $1000^{\circ} \mathrm{C}$ under ambient atmosphere. The weight loss $(4 \%)$ at about $66^{\circ} \mathrm{C}$ may be attributed to the evaporation of adsorbed water. There is a weight loss of $24 \%$ at approximately $344^{\circ} \mathrm{C}$ which may be connected with decomposition of nickel hydroxide. XRD pattern of the heattreated sample is given in Figure 5(a). All of detectable peaks are consisted with those of $\mathrm{NiO}$ phase. TEM results in Figure 5(b) indicated $\mathrm{NiO}$ nanocrystals with a length of $50 \mathrm{~nm}$ and a width of $10-25 \mathrm{~nm}$. The appearance of partial diffraction spots in selected electron diffraction image (Figure 5(c)) indicated the improvement of $\mathrm{NiO}$ crystallinity. In order to further compare our experiment, we performed the reduction of $\mathrm{NiO}$ powders in $\mathrm{H}_{2}$ atmosphere at high temperature. XRD analysis of the final samples confirmed cubic nickel particles, whose sharp peaks indicated further growth of $\mathrm{NiO}$ after high temperature treatment.

When we choose monodispersed silica nanospheres or commercial silica nanospheres as templates to synthesize nickel-silica hollow spheres, the process of reaction and properties of samples will alter greatly, which probably attributes to the mutual interaction between catalyst and silica templates [15]. Prokes et al. [16] pointed out that the concentration of $\mathrm{SiOH}$ groups on surface of silica particles can influence bonding of metal complexes to the surface, and the subsequent morphology of a created, decorated metal ion surface. Hereon, we mainly discuss the as-prepared silica templates' function in surface deposition and prosperities of final samples. 


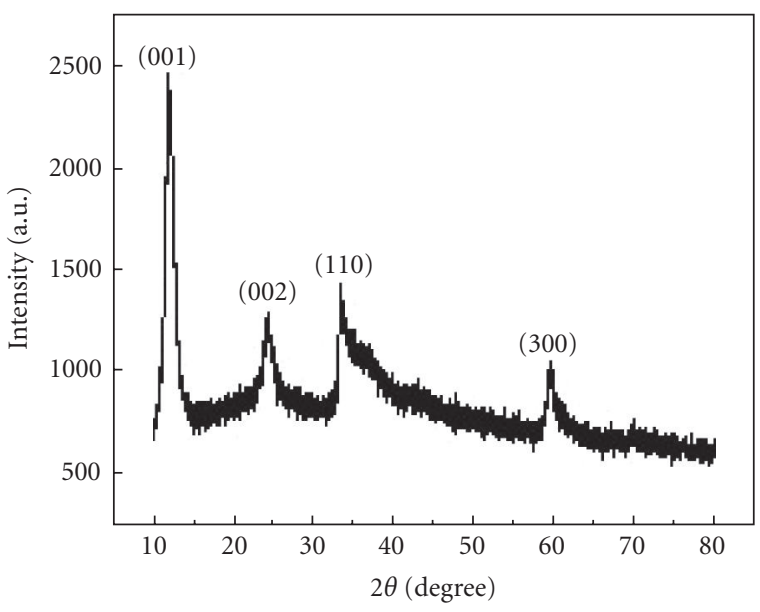

(a)

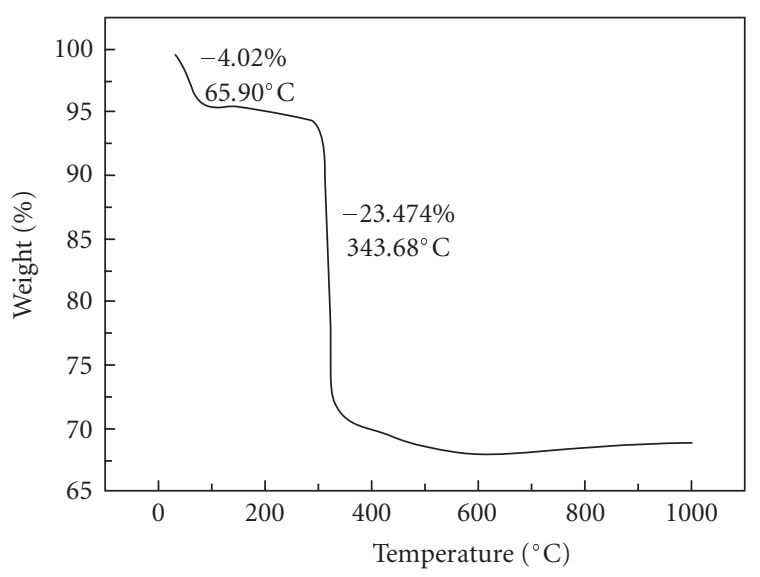

(b)

FIGURE 4: XRD profile (a) and thermogravimetric curve (b) of precipitates obtained from decomposing urea in nickel nitrate solution without any silica nanospheres.

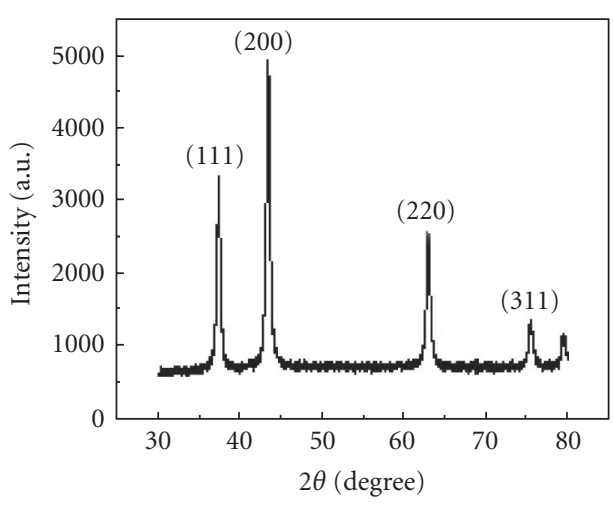

(a)

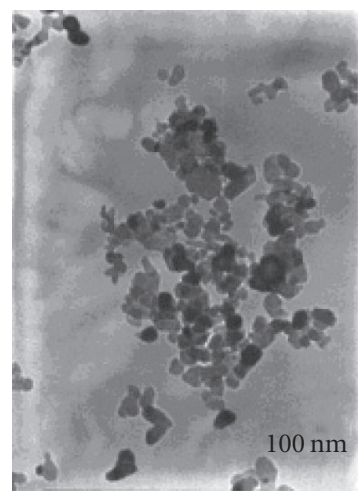

(b)

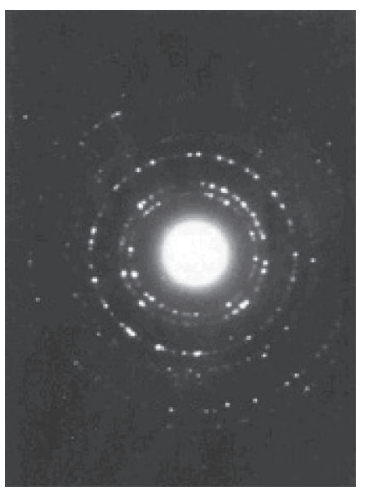

(c)

Figure 5: XRD pattern (a), TEM image (b), and selected electron diffraction pattern (c) of heat-treated samples prepared from Ni(OH) ${ }_{2}$.

We used the homogeneous precipitation [15] of slowly decomposing urea at above $70^{\circ} \mathrm{C}$ in nickel nitrate solution to coat sediments on the surface of silica. With the presence of silica spheres in the solution system, hydrosilicate will form through reaction between $\mathrm{Ni}(\mathrm{OH})_{2}$ and $\mathrm{SiO}_{2}$. The merit of selecting homogeneous precipitation is that nickel particles have an excellent colloidal dispersion which may influence the activity and selectivity of acetone hydrogenation. This preparation method also considerably affects the thermal stability of crystal particles, because sintering growth of crystal particles mainly depends on the initial-dimensional dispersion of crystals.

The as-prepared silica nanospheres in our experiment on nano-scale can easily react with nickel ion or $\mathrm{Ni}(\mathrm{OH})_{2}$ by precipitating on the surface of silica particles compared to commercial silica nanospheres on micron scale, due to the better solubility and higher concentration of hydroxyl groups on the surface of silica nanospheres. In the experiment, nucleation and deposition only occur on the surface of silica nanospheres rather than in solution system based on the actual reaction phenomenon. First, if deposition takes place in solution system, final samples inevitably consist of the separate $\mathrm{Ni}(\mathrm{OH})_{2}$ sediments and the shells of $\mathrm{Ni}(\mathrm{OH})_{2}$ on surface of silica. However, under observation of electronic microscope, except the abundant core-shell particles, it does not contain any separate $\mathrm{Ni}(\mathrm{OH})_{2}$ particle with size of 25 $50 \mathrm{~nm}$, which appeared in the control experiment, the solo preparation of $\mathrm{Ni}(\mathrm{OH})_{2}$ without any silica nanosphere as discussed above. The possible explanation is that the continuous dissolved silica or hydroxyl groups on surface of nanospheres react with nickel ion eventually form indissoluble nickel hydrosilicate to adhere to the spherical particles. From TEM image (Figure 6), we can obviously observe the shell structure, which is accumulated by nanoparticles, totally different from the smooth surface of silica before coating. We also found that the shell with some thickness gradually separates from the sphere as a result of fuzz on the shell. The thickness of shell and the length of fuzz increase correspondingly with 


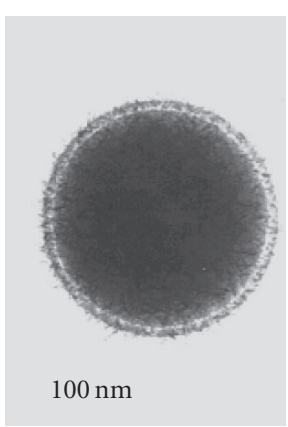

(a)

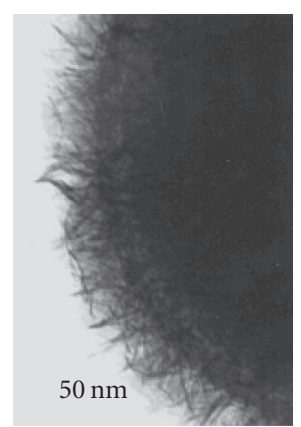

(b)
FIGURE 6: TEM photograph of silica/nickel hydrosilicate core-shell structure.

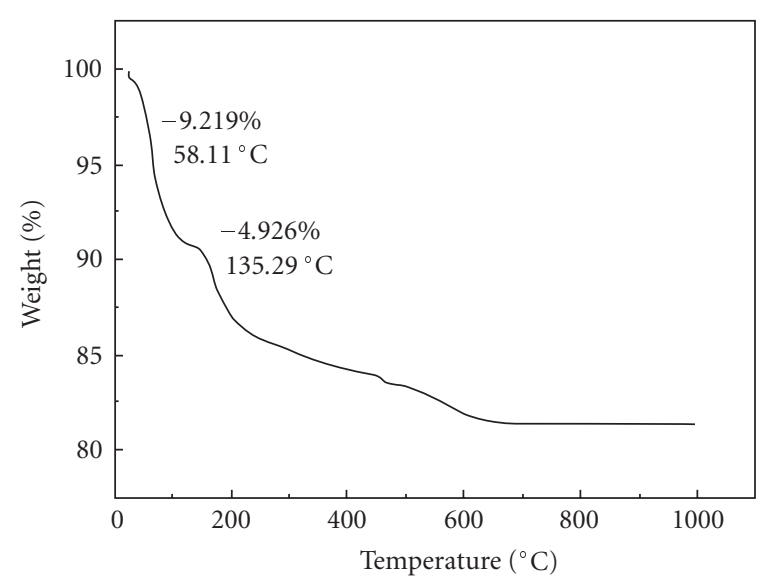

Figure 7: Thermogravimetric curve of silica/hydrosilicate coreshell particles.

the times of coating. In addition, the chemical composition of the shell structure is not a simple $\mathrm{Ni}(\mathrm{OH})_{2}$ but nickel hydrosilicate, XRD analysis of the heated composite core-shell spheres indicates no obvious physical structure alteration of the shell.

Figure 7 shows the thermal decomposition result of silica/hydrosilicate core-shell particles under ambient atmosphere. The weight loss of $9 \%$ at about $58^{\circ} \mathrm{C}$ and $5 \%$ at approximately $135^{\circ} \mathrm{C}$ may be associated to water desorption on silica. However, the decomposition of nickel hydroxide at $343^{\circ} \mathrm{C}$ is not obvious in Figure 8, probably because of the thermal stability of nickel hydrosilicate at this temperature.

Besides, the more important influence is that core-shell nanostructures using, the as-prepared silica nanospheres are less reducible, compared with simplex nickel hydroxide or commercial silica supported samples. We have carried out the deoxidization of silica/nickel hydrosilicate composite spheres through pure $\mathrm{H}_{2}$, which could not be completely reduced even at $550^{\circ} \mathrm{C}$. However, after removing silica cores, the shells are easier to be reduced even at $450^{\circ} \mathrm{C}$. The expla-

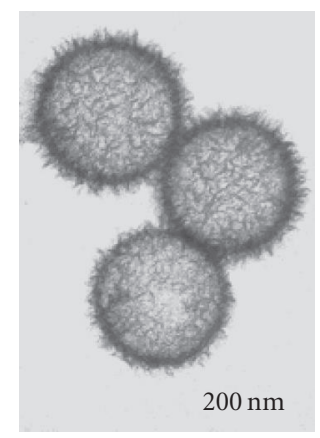

(a)

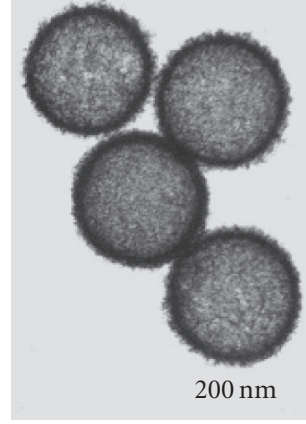

(b)
FIGURE 8: TEM images of nickel-silica hollow nanospheres (a) reduction after removing silica cores, (b) removing silica cores after reduction [12].

nation is that the low interaction between silica and nickel or nickel ion [12]. In order to weaken the interaction between shell and silica sphere, reduce the shell more completely and keep the porous surface, we prepared nickel-silica hollow spheres by removing cores before the process of deoxidization.

Our previous work [12] showed the distinctive morphology of nickel-silica hollow nanospheres through different sequences of reduction and etching (Figure 8). From Figure 8(a), we observe that preparing nickel-silica hollow nanospheres by reduction after removing silica cores reserves the fuzz structure of the shell and produces large specific surface area, which implies the potential application in gas heterogeneous catalysis. Compared to the former samples, the fuzz structure (Figure 8(b)) on the surface of nickel-silica hollow nanospheres prepared by removing silica cores after reduction has been destroyed, the shell also becomes denser. No matter the sequence of etching and reduction, both involved the process of etching silica cores. In order to keep the morphology of hollow spheres, we should control the concentration of base solution as shown in Figure 9(b), otherwise the shape of hollow sphere is distorted or broken up, even collapse as shown in Figure 9(a).

Consequently, considering the efficiency of reduction, the morphology, and property of samples, we should choose the way of preparing nickel-silica composite hollow spheres by removing silica cores before reduction.

\subsection{Acetone hydrogenation using nickel-silica hollow nanospheres as catalyst}

Recent works have been carried out on activity and selectivity of nickel-silica hollow nanospheres in acetone hydrogenation reaction with resultant samples typically 2-propanol, methyl isobutyl ketone (MBIK), methyl isobutyl carbinol (MIBC), and disopropyl ether (DIPE). Unnikrishnan and Narayanan [17] have reported superior $\mathrm{Ni} / \mathrm{Al}_{2} \mathrm{O}_{3}, \mathrm{Co} / \mathrm{Al}_{2} \mathrm{O}_{3}$, and layered double hydroxides or hydrotalcite-like compounds such as $\mathrm{NiMgAl}$, CoMgAl to prepare 2-propanol, and MBIK with high selectivity and conversion at a relatively 


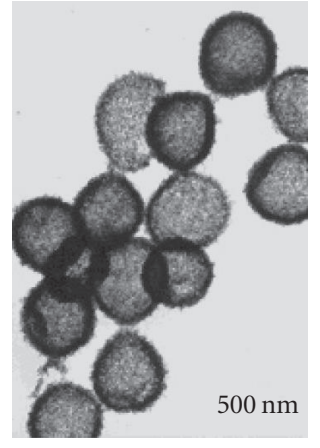

(a)

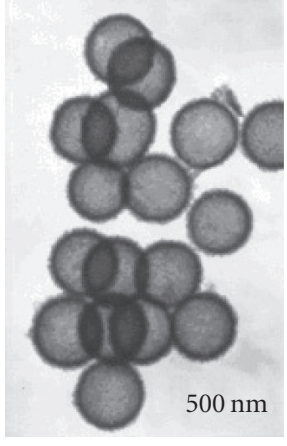

(b)
FIGURE 9: TEM images of samples from choosing different concentrations of $\mathrm{NaOH}$ to remove silica cores: (a) $\mathrm{NaOH} 5 \mathrm{~mol} / \mathrm{L}$; (b) $\mathrm{NaOH} 0.5 \mathrm{~mol} / \mathrm{L}$.

low temperature of $100^{\circ} \mathrm{C}$. Even though $\operatorname{Ir}(0)_{\mathrm{n}}$ nanoclusters plus $\mathrm{HCl}$ from Saim Özkar's recent work [18] can achieve selectivity of $95 \%$ to 2-propanol with nearly $100 \%$ acetone conversion at low temperature of $22^{\circ} \mathrm{C}$, the main drawback is the limited catalytic lifetime due to nanocluster precipitation. While in our work, nickel-silica hollow nanospheres show relative high conversion of $70 \%$ and selectivity of $82.7 \%$ to 2-propanol and $13.8 \%$ to MBIK. Compared to the Gandia et al. [13] work using silica-supported nickel as catalyst at $200^{\circ} \mathrm{C}$ and atmosphere pressure, the major difference is higher acetone conversion, less selectivity to 2-propanol, and with some amount of MBIK samples in our catalyst system. The nickel-silica hollow nanospheres show lower selectivity to 2-propanol, mainly due to relatively high activity of this catalyst for the formation of MBIK associated with the special structure of catalyst.

\section{EXPLANATION OF THE MECHANISM OF ACETONE HYDROGENATION REACTION IN OUR CATALYST SYSTEM}

It has been reported that using pure nickel particles or bare silica spheres as catalyst to perform acetone hydrogenation cannot produce MBIK. Consequently, the synthesis of MBIK in nickel-silica hollow sphere catalyst system is likely performed on the layer between nickel and silica. Narayanan and Unnikrishnan [19] have pointed out that acetone conversion takes place both on metal sites and on acidic or basic sites of catalyst, but hydrogenation of acetone to 2-propanol over a metal site is more favorable than condensation of acetone over an acid-base site. In their researches on metal containing layered double hydroxides efficient catalyst, Unnikrishnan and Narayanan [17] indicated that the formation of MIBK or MIBC is attributed to the cooperative effect of both hydrogenating and acid-base properties of catalyst. Through detailed examination of Unnikrishnan's research, we found out that nickel-silica hollow sphere catalytic mechanism is very similar to what Unnikrishnan has pointed out. On the one hand, the relative high activity and conversion of hollow nanospheres in acetone hydrogenation can be explained by the following factors. (1) Nickel-silica hollow nanospheres with high BET surface areas $\left(288 \mathrm{~m}^{2} / \mathrm{g}\right)$ can absorb larger amount of $\mathrm{H}_{2}$ than normal nickel particles [12]. In addition, the porous structure would be favorable for transportation of $\mathrm{H}_{2}$ and make it likely stay in the inner part of hollow spheres to prolong the reaction time. (2) The shells are composed of acicular nanoparticles with an average size of $15 \mathrm{~nm}$. For the structure-sensitive reaction, size of particles can greatly influence the selectivity and activity. Furthermore much larger amount of acetone can be absorbed on surface of nickel particles. There are two mechanisms to describe acetone absorption which both involve charge transfer, one is that $\pi$ electrons are transferred from carbonyl group to free $\mathrm{d}$ orbital of the metal, and the other involves d electrons of nickel filling in the antibonding orbital of carbonyl group. As Rao et al. [20] pointed out, either way of charge transfer can weaken or break the bond of carbonyl group and make the reaction easier. Consequently, the unique porous structure with high capacities of $\mathrm{H}_{2}$ and acetone absorption and low energy for acetone hydrogenation contributes to the high activation and conversion. On the other hand, nickel-silica hollow spheres contain a nickel-silica interface where electron deficient sites such as nickel ion resulting from the unreduced $\mathrm{Ni}(\mathrm{OH})_{2}$ or nickel hydrosilicate [12]. At meantime, silica can act as base site to attract a methyl proton and $\mathrm{Ni}^{\mathrm{n}+}$ can activate carbonyl group of acetone when $\mathrm{O}$-atom of carbonyl coordinates to the $\mathrm{Ni}^{\mathrm{n}+}$ site. As a result, absorbed methyl reacts with the neighboring coordinated carbonyl and neighboring $\mathrm{H}$ atoms absorbed on the metal sites to form MIBK and water.

Based on the fact that the main composition of nickelsilica hollow spheres is nickel metal, and hydrogenation of acetone to 2-propanol over a metal site is more favorable than condensation of acetone over an acid-base site as we discussed above, the acetone hydrogenation reaction shows the higher selectivity to 2-propanol and lower selectivity to MBIK and MBIC.

\section{CONCLUSIONS}

The above researches illustrate that monodispersed silica nanospheres can be used as templates for large-scale preparation of nickel-silica composite hollow spheres with an outer diameter of $650 \mathrm{~nm}$ and a thickness of $40 \mathrm{~nm}$ through encapsulating and etching. The nickel-silica hollow spheres display relative high activity and selectivity in acetone hydrogenation, showing potential applications as nanocatalysts. It is suggested that the unique porous structures with high capacities of $\mathrm{H}_{2}$ and acetone absorption and lower energy for acetone hydrogenation contribute to the high activity and conversion. The synthesis process might be extended to prepare hollow nanospheres of other materials for catalytic application.

\section{ACKNOWLEDGMENT}

This work was supported by the Natural Science Foundation of China (20321101, 20125103, and 90206034). 


\section{REFERENCES}

[1] K. J. Klabunde, "Introduction to the nanoworld," in Nanoscale Materials in Chemistry, K. J. Klabunde, Ed., pp. 6-7, John Wiley \& Sons, New York, NY, USA, 2001.

[2] J. Parker, "Applications of nanocrystals," in Nanoscale Materials in Chemistry, K. J. Klabunde, Ed., pp. 280-285, John Wiley \& Sons, New York, NY, USA, 2001.

[3] W. E. Buhro and V. L. Colvin, "Semiconductor nanocrystals: shape matters," Nat Mater, vol. 2, no. 3, pp. 138-139, 2003.

[4] E. Matijevic, "Controlled colloid formation," Current Opinion in Colloid \& Interface Science, vol. 1, p. 176, 1996.

[5] A. Polman and P. Wiltzius, "Materials science aspects of photonic crystals," MRS Bulletin, vol. 26, no. 8, pp. 608-610, 2001.

[6] P. Jiang, J. Cizeron, J. F. Bertone, and V. L. Colvin, "Preparation of macroporous metal films from colloidal crystals," Journal of the American Chemical Society, vol. 121, no. 34, pp. 7957-7958, 1999.

[7] W. Wang, B. Gu, L. Liang, and W. Hamilton, "Fabrication of two- and three-dimensional silica nanocolloidal particle arrays," Journal of Physical Chemistry B, vol. 107, no. 15, pp. 3400-3404, 2003.

[8] K. R. Peters, G. Rutter, H. H. Gschwender, and W. Haller, "Derivatized silica spheres as immunospecific markers for high resolution labeling in electron microscopy," Journal of Cell Biology, vol. 78, no. 2, pp. 309-318, 1978.

[9] X. Q. Liu, J. M. Xing, Y. P. Guan, G. B. Shan, and H. Z. Liu, "Synthesis of amino-silane modified superparamagnetic silica supports and their use for protein immobilization," Colloids and Surfaces A: Physicochemical and Engineering Aspects, vol. 238, no. 1-3, pp. 127-131, 2004.

[10] D. Wang, R. A. Caruso, and F. Caruso, "Synthesis of macroporous titania and inorganic composite materials from coated colloidal spheres-a novel route to tune pore morphology," Chemistry of Materials, vol. 13, no. 2, pp. 364-371, 2001.

[11] K. M. Kulinowski, P. Jiang, H. Vaswani, and V. L. Colvin, "Porous metals from colloidal templates," Advanced Materials, vol. 12, no. 11, pp. 833-838, 2000.

[12] P. Jin, Q. Chen, L. Q. Hao, R. F. Tian, L. X. Zhang, and L. Wang, "Synthesis and catalytic properties of nickel-silica composite hollow nanospheres," Journal of Physical Chemistry B, vol. 108, no. 20, pp. 6311-6314, 2004.

[13] L. M. Gandia, A. Diaz, and M. Montes, "Selectivity in the hightemperature hydrogenation of acetone with silica-supported nickel and cobalt catalysts," Journal of Catalysis, vol. 157, no. 2, pp. 461-471, 1995.

[14] W. Stobber, A. Fink, and E. Bohn, "Controlled growth of monodisperse silica spheres in the micron size range," Journal of Colloid and Interface Science, vol. 26, pp. 62-69, 1968.

[15] J. T. Richardson and R. J. Dubus, "Preparation variables in nickel catalysts," Journal of Catalysis, vol. 54, no. 2, pp. 207218, 1978.

[16] S. M. Prokes, W. E. Carlos, L. Seals, S. Lewis, and J. L. Gole, "Formation of ferromagnetic $\mathrm{Ni} / \mathrm{SiO}_{2}$ nanospheres," Materials Letters, vol. 54, no. 1, pp. 85-88, 2002.

[17] R. Unnikrishnan and S. Narayanan, "Metal containing layered double hydroxides as efficient catalyst precursors for the selective conversion of acetone," Journal of Molecular Catalysis A: Chemical, vol. 144, no. 1, pp. 173-179, 1999.

[18] S. Özkar and R. G. Finke, "Iridium(0) nanocluster, acidassisted catalysis of neat acetone hydrogenation at room temperature: exceptional activity, catalyst lifetime, and selectivity at complete conversion," Journal of the American Chemical Society, vol. 127, no. 13, pp. 4800-4808, 2005.
[19] S. Narayanan and R. Unnikrishnan, "Selective hydrogenation of acetone to methyl isobutyl ketone (MIBK) over coprecipitated $\mathrm{Ni} / \mathrm{Al}_{2} \mathrm{O}_{3}$ catalysts," Applied Catalysis A: General, vol. 145, no. 1-2, pp. 231-236, 1996.

[20] R. S. Rao, A. B. Walters, and M. A. Vannice, "Influence of crystallite size on acetone hydrogrnation over copper catalysts," Journal of Physical Chemistry B, vol. 109, no. 6, pp. 2086-2092, 2005. 

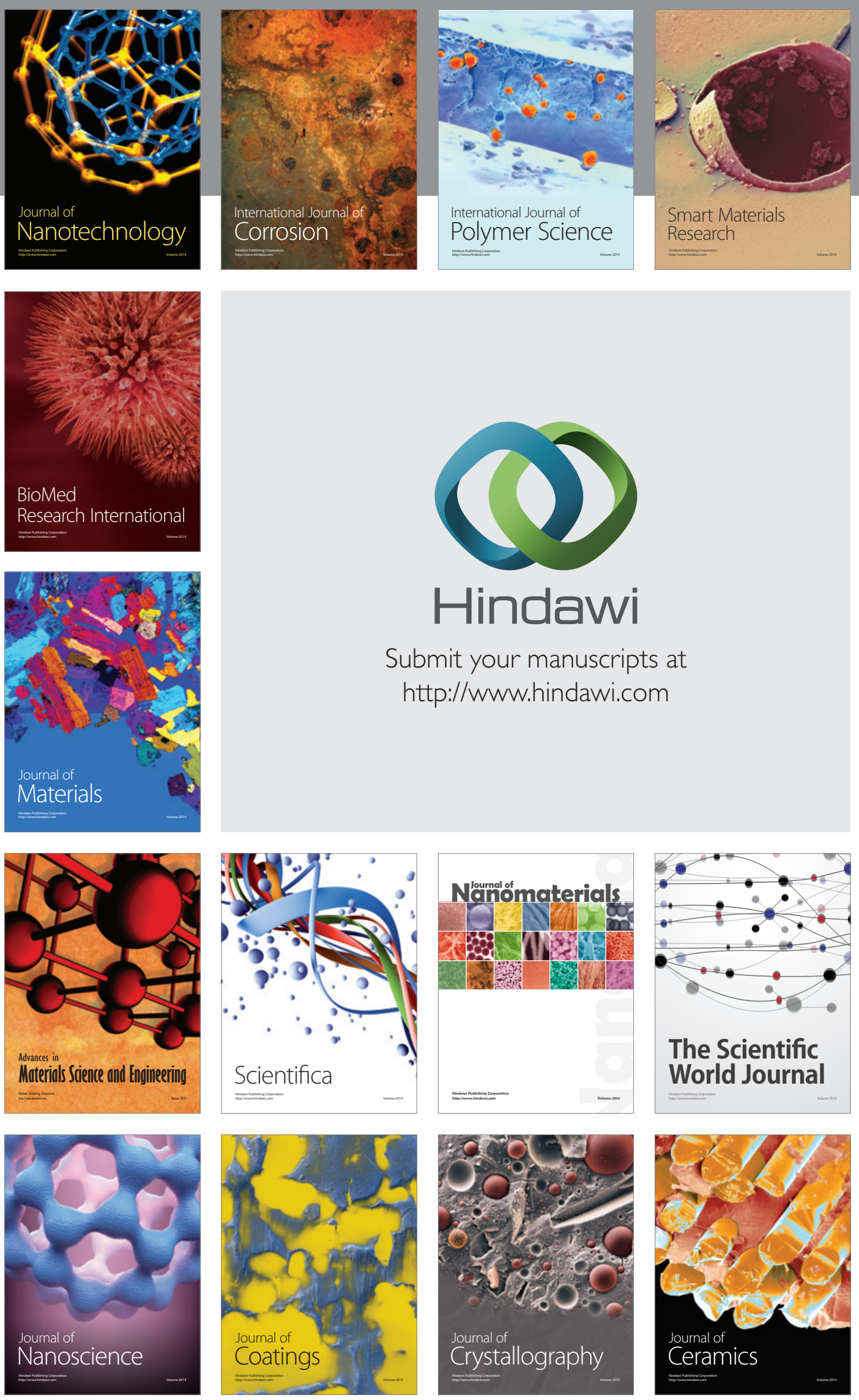

The Scientific World Journal

Submit your manuscripts at

http://www.hindawi.com

\section{World Journal}

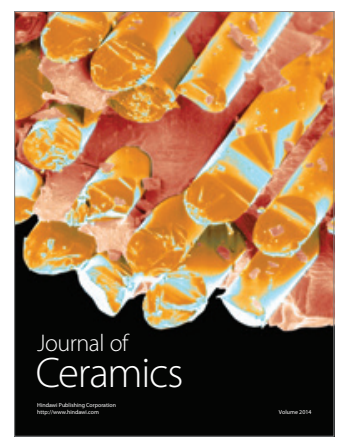

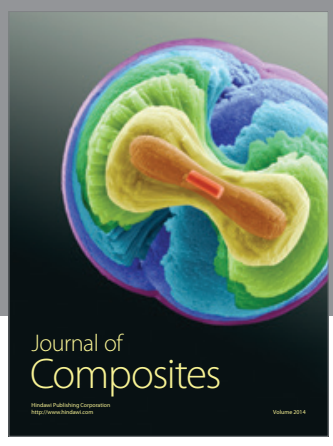
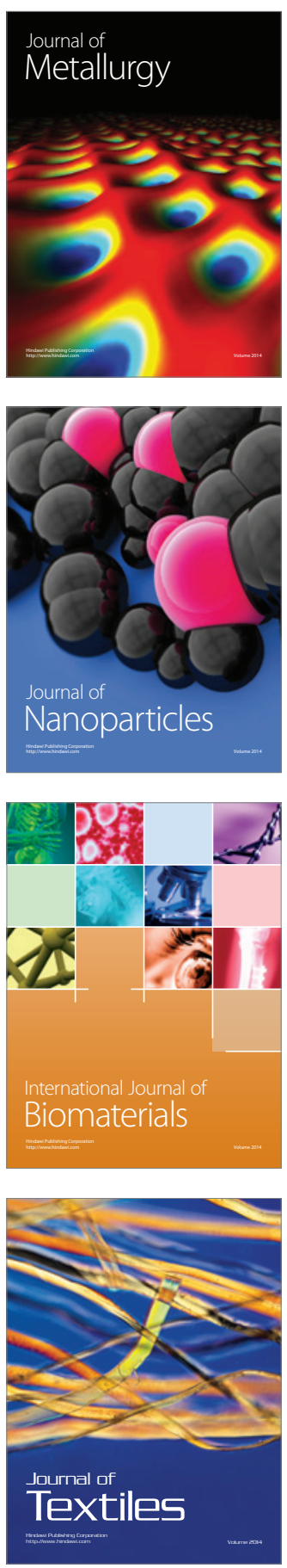